\title{
Application value of quantitative system pharmacology in drug discovery for traditional Chinese medicine
}

\author{
Fuxue Meng $^{1^{*}} \mid$ Xiaomai Tao ${ }^{2}$
}

\author{
${ }^{1}$ Department of Hematology and \\ Rheumatology, The Third \\ Affiliated Hospital of Guizhou \\ Medical University, Duyun, \\ Guizhou, China \\ ${ }^{2}$ College of Biology and \\ Engineering, Guizhou Medical \\ University, Guiyang, Guizhou, \\ China
}

\begin{abstract}
Quantitative system pharmacology (QSP) is a discipline that combines computational models of systems biology and systems pharmacology. With the development of high-throughput genomics techniques (genomics, transcriptomics, proteomics, and metabolomics) as well as computer and bioinformatics methods, systems biology and systems pharmacology modeling are widely used to comprehend human biology and disease progression, predict the effectiveness and safety of drug candidates. Due to the advancement of big data and high-quality database, the application of QSP, especially the pre-clinical stage that guides early drug discovery, is increasingly widespread. The traditional drug discovery process takes a long time yet has a low success rate. The early intervention and full participation of QSP in the development of new drugs discovery can form a model-led drug development model to improve the efficiency of drug discovery and scientific appraise, reduce the cost of research and development, and shorten the time to market for new drugs. This article reviews the differences between QSP and other quantitative pharmacology, the problems faced by traditional Chinese medicine research, and the value of QSP in traditional Chinese medicine research, with a view to providing reference and support for the research and development of new traditional Chinese medicine.

Keywords: Quantitative system pharmacology, Traditional Chinese medicine, Drug discovery
\end{abstract}

\section{1 | INTRODUCTION:}

A series of omics disciplines including Genomics, Proteomics, Metabolomics, and recently developed Interactomics, Disomomics, and Zhenggomics developments have brought profound changes to modern medicine and drug discovery as well as development ${ }^{[1,2]}$.
On the one hand, high-throughput sequencing technology, microarrays, two-way gel experiments, large-scale mass spectrometry, NMR spectroscopy and other technologies provide unprecedented technical support for drug target discovery. On the other hand, the wide application of various highthroughput technologies has also exposed scientists to the increasing storage, retrieval, analysis, and 
interpretation of massive data. To cope with the "data explosion" and solve complex biological problems, medical scientists, biologists, and pharmacists are constantly seeking help from mathematics and computer experts. As a result, a number of disciplines based on computing and simulation, such as bioinformatics, computational biology, pharmaceutical informatics, systems biology, network pharmacology and systems pharmacology, quantitative pharmacology and quantitative system pharmacology (QSP) came into being ${ }^{[3,4]}$.

System pharmacology is a new conceptual framework that simulates traditional pharmacological parameters. Given the pharmacology and pharmacokinetics, the simulation and calculation framework of system medicine is run in experiments ${ }^{[5]}$. Under a unified time-space multi-scale framework, from different levels of molecules, cells, tissues, organs, individuals, groups and environments, to study the mechanism of changes in the body's functions when drugs are used to treat diseases, and establish the role of drugs for the body Interactions and interrelationships between micro to macro levels ${ }^{[6]}$. Quantitative pharmacology uses modeling and simulation (M\&S) technology to quantify information such as pharmacokinetics (PK), pharmacodynamics (PD), body function, disease mechanism, and test progress. A subject of chemical research ${ }^{[7,8]}$. Through various quantitative methods and methods, the various aspects of drug disposal and function and the important factors affecting these processes are highly conceptualized and abstracted, and mathematical models are established and simulated through strict logical reasoning. This research can rationally and systematically integrate drug and product-specific information and biological system-specific information, discover and establish new laws, and use

Supplementary information The online version of this article (https://doi.org/10.15520/mcrr.v3i9.113) contains supplementary material, which is available to authorized users.

Corresponding Author: Fuxue Meng

Department of Hematology and Rheumatology, The Third Affiliated Hospital of Guizhou Medical University, Duyun, Guizhou, China

Email:mfx5675143@126.com these laws to formulate drug development strategies to improve clinical research decision-making efficiency and optimize treatment plans. It continuously absorbs nutrition from the disciplines of clinical pharmacology, biostatistics, pharmacokinetics, registration science, clinical pharmacy, medicine and computer programming, and reorganizes the knowledge system. It has developed into an interdisciplinary with certain characteristics so far ${ }^{[9]}$. Quantitative system pharmacology (QSP) is committed to the development and utilization of mathematical models, and on the basis of combining experiments to discover small molecules, confirm targets, study disease pathogenesis and treatment mechanisms to improve drug efficacy and reduce toxicity Side effects, eventually achieving the precise control of complex intracellular networks and changing the pathophysiology of new drugs. With the development of high-throughput omics technology and computer and bioinformatics methodologies, systems biology and systems pharmacology modeling methods are used to understand human biology and disease progression $^{[10]}$ and to predict the effectiveness of drug candidates And security ${ }^{[11]}$. With the advancement of big data and high-quality knowledge base, the application of QSP, especially the pre-clinical stage that guides early drug development, is increasingly widespread ${ }^{[12]}$.

\section{2 | COMPARISON OF QSP AND OTHER QUANTITATIVE PHARMACOLOGY:}

QSP is one of quantitative pharmacology. Quantitative pharmacology can be divided into: population PK/PD model ( population pharmaco-kinetics/ pharmacodynamics, PPK/PD ), physiologically-based pharmacokinetics (PBPK) $)^{[13]}$, model-based metaanalysis (model-based meta analysis, MBMA) ${ }^{[14]}$ and quantitative systems pharmacology (QSP) ${ }^{[15]}$ and other specific research methods. After decades of development, the quantitative pharmacology research model has evolved from pure PK mathematical modeling and simulation ${ }^{[16]}$ to a learn-confirm cycle model, and will be updated iteratively ${ }^{[17]}$. Quantitative pharmacology research process is the process of modeling and simulation. The applica- 
tion of modeling and simulation technology in the Chinese pharmaceutical industry is relatively limited, but it has been widely used abroad, including from micro-structure-effect relationship evaluation to macro-new drug development costs Benefit prediction, from candidate compound screening to postmarketing business strategy recommendations, includes modeling and simulation applications recommended by various regulatory guidelines ${ }^{[18,19]}$ and industry management codes ${ }^{[20,21]}$.

Compared with traditional pharmacological models, QSP has the unique feature of mechanically integrating key molecules into the biological network of specific targets of drug candidates, and qualitatively and multi-scalely compare them to the pharmacodynamic markers of target diseases Combining biomaterials and clinical terminals. Therefore, QSP can simultaneously predict multiple clinical responses, assist in the first human trial of dose selection, and predict test results and iterative model calibration, prediction, and verification ${ }^{[22]}$. At the same time, drug discovery pipelines based on biomarkers and target identification based on signaling pathways can achieve accurate drug development. It must be emphasized that QSP is basically developed from existing complementary modeling methods and still benefits from existing complementary modeling methods, including systems biology, PKPD and PBPK modeling methods. With the advancement of computing methods, the acquisition of new data and the development of more biological knowledge, the natural development of some drug discovery problems is toward a more mechanized (perhaps holistic) description of the system, allowing the data collected Extrapolation through QSP modeling outside the set and solve new problems ${ }^{[23]}$.

Through years of drug development and implementation, PKPD modeling has proven to be of great value in clarifying the pharmacokinetics of therapeutic interventions and the resulting pharmacodynamic effects ${ }^{[24]}$. PKPD parameters derived from relevant preclinical studies and appropriately adjusted according to clinical conditions enable prospective simulations to assess key drug development issues, such as clinical dose levels and frequency ${ }^{[25,26]}$. Over the years, translational PKPD modeling has surpassed empirical models and combined more me- chanical components to build a mechanism-based PKPD model, thereby facilitating bio-driven translation across species or different patient groups. The trend of natural evolution from empirical PKPD to mechanical PKPD to QSP came into being. However, as the original QSP white paper emphasized that this mixed complementary view has always existed $^{[27]}$. The development of QSP aims to meet the possibility that people hope to incorporate other biological mechanisms into it, and at the same time has the potential to characterize these important biological signals together.

The PBPK model is another model that is often debated as to whether it belongs to the QSP model definition. Erica L. Bradshaw and his team made a good analysis of this issue as follows ${ }^{[28]}$ : Similar to the evolution of PKPD to QSP, the mechanical PBPK model shows that a highly mechanical model can provide predictive biological insights and provide the pharmaceutical industry with Bring value and lay the foundation for the QSP model. Although the PBPK model may have important mechanistic details and depend on system and drug-dependent parameters, the focus of these two modeling methods is to separate pharmacodynamics and disease biology/(pathology) physiological components.

The PBPK model focuses on absorption, distribution, metabolism, excretion, and pharmacokinetic issues, while the QSP model focuses on regulating a given target and its subsequent effects on basic biology and/or disease pathology. Therefore, the mechanism PBPK model may be more suitable for the quantitative system pharmacokinetic model (QSPK) rather than the QSP model. Although the main focus of the two modeling methods may be different, it should be emphasized that these methods are not exclusive and may require the PBPK model to be linked to the QSP model, for example, to drive target tissue-specific drug concentrations.

In fact, throughout the entire pharmacological proof process and all relevant decision-making steps, multimodal exploratory and predictive results can be incorporated into a single prediction model ${ }^{[29]}$. In conclusion, QSP modeling has been used throughout the preclinical drug development process to explore the therapeutic and toxic effects of drugs in the 
entire therapeutic field, including: metabolism ${ }^{[30]}$, autoimmune diseases ${ }^{[31]}$, tumors ${ }^{[32,33]}$, Neuroscience and other fields ${ }^{[34]}$. And it has been proved to improve the drug development efficiency and scientific decision-making level in various diseases ${ }^{[35]}$. This article analyzes the value of QSP in the research and development of new Chinese medicines with a view to providing support for the research and development of new Chinese medicines.

\section{3 | THE VALUE OF QSP IN THE RESEARCH AND DEVELOPMENT OF CHINESE MEDICINE:}

The human body is a complex biological network system, including signal network, metabolic network, protein interaction gene regulation network and so on. If the disease is considered to be a change in the body's original network balance, then the purpose of the drug is to enable the body to return to its original equilibrium health. Due to the complexity and stability of the biological network, the operation is usually adjusted for multiple nodes (targets) in the network system at the same time, in order to achieve the desired therapeutic effect. In fact, existing studies have shown that [36], drugs and targets of action tend to form an enriched network, rather than a one-to-one correspondence. Most drugs work through multiple targets, and about half of the drug targets are related to multiple diseases, so that complex cross-networks are formed between drugs and disease genes ${ }^{[37]}$. Interestingly, Chinese medicine is just a multi-component, multi-target synergy system that meets this requirement. As a system, Chinese medicine has many similarities with the philosophy of system biology. For example, traditional Chinese medicine is considered to be a multi-target system, however, the multi-target technology of traditional Chinese medicine is not based on the molecular (gene, protein, etc.) level, but on the systematic thinking or philosophical methods. It emphasizes the establishment of mathematical models to study the interaction between the system's macroscopic functions, changes and other system behaviors, and microscopic genetic modification and biological network regulation $^{[38]}$. In fact, how to directly apply western system theory to Chinese medicine research still has great difficulty in methodology.

With the rapid development of the global pharmaceutical economy, China's pharmaceutical industry is facing huge challenges, especially the $R \& D$ of Chinese medicine has ushered in unprecedented opportunities. As the cost of research and development of chemical synthetic drugs is increasing, the cycle is prolonged, the success rate is greatly reduced, and the environmental pollution caused is becoming more and more serious. The interest in traditional medicine is also increasing ${ }^{[39]}$. At present, the research and development of new drugs of traditional Chinese medicine has become a popular research direction in the international pharmaceutical industry. However, the ingredients of traditional Chinese medicine and its compound are extremely complex, and the pharmacological effects are also diverse ${ }^{[39,40]}$. Based on this, although the research on the ingredients of traditional Chinese medicine has a history of hundreds of years, the huge bottleneck restricting the development of traditional Chinese medicine is still manifested in core issues such as unclear material basis and unknown mechanism of action $^{[41]}$. Inheritance and development in China has seriously hampered the move of Chinese medicine to a larger international stage. Because of its complex composition and huge system, it is extremely difficult to study the effect of traditional Chinese medicine on the body from the mixture system. It is because the process of developing new drugs is very complicated, often involving multi-disciplinary comprehensive applications $^{[42,43]}$. Therefore, it is urgent to find an effective drug research method.

Classical pharmacology has played an extremely important role in drug development. Traditionally, animals have been used to carry out drug activity tests, and further study of the target organs of drug action and their toxicity ${ }^{[44-46]}$. However, it is often extremely difficult to build a good disease model animal. This problem is particularly prominent in the research of traditional Chinese medicine. For example, the cold and heat of human diseases and the deficiency and reality are difficult to reproduce on animal models ${ }^{[47]}$. Traditional Chinese Medicine emphasizes the differentiation of syndromes, the use 


\section{MANUSCRIPT CENTRAL}

of compound prescriptions and compatible medications. Compound prescription of traditional Chinese medicine is the specific manifestation of the essence of dialectical theory of traditional Chinese medicine, and compatibility is the key to the effect of prescription ${ }^{[48]}$. However, the process of making traditional Chinese medicine lacks a systematic drug discovery process similar to Western medicine. Therefore, in-depth research on traditional Chinese medicine will often face the following problems ${ }^{[49]}$ : (1) Traditional Chinese medicine theory mainly understands the clinical effectiveness and safety of traditional Chinese medicine from a collation and macro perspective, lacking surprise process analysis and monitoring, drug efficacy or toxicity The effect mechanism is also lacking in-depth study. (2) The origin of the medicinal materials is different, and the combination of the combination of them has a large variability, and the quality is difficult to be effectively controlled. (3) Different compatibility determines the different absorption, distribution, metabolism, excretion and toxicity properties of medicinal components. (4) The effective ingredients of many traditional Chinese medicines are not yet clear, and the material basis of the efficacy and toxicity has not been completely resolved. (5) Because traditional Chinese medicine is mainly oral and external administration, biotransformation occurs through the gastrointestinal tract and the body, and the quantitative and qualitative changes of the effective components in the body will lead to differences in its efficacy and attending symptoms. There is a lack of basic data on how these changes affect drug efficacy and safety. (6) Chinese medicine is a multi-component, multi-target system. It is generally believed that synergy or antagonism will occur between multiple active ingredients of traditional Chinese medicine, but it is extremely difficult to study the synergistic interaction between components.

Systems pharmacology integrates systems biology and pharmacology methods to promote new drug development and understanding of herbal treatment mechanisms. As the core driving force of medical development in the 21 st century, systemic pharmacy is a dialectic medicine based on the principles and methods of modern systems theory and based on systems biology. Systematic pharmacological analysis usually relies on multiple variables at the genomic level to construct networks, evaluate drug behavior, and promote people's understanding of treatment mechanisms. By linking pathways and networks, systematic pharmacology also ensures the accuracy of pharmacokinetic and pharmacodynamic prediction models. It provides a complete and multi-scale systematic research method for the acquisition of herbal active molecules, the acquisition of targets, the analysis of the nature of target networks, and the relationship between targets and diseases ${ }^{[50,51]}$. Using systematic pharmacological methods to study traditional Chinese medicine can help us clarify the theory of traditional Chinese medicine on the whole, level and information level, establish an operational platform for the prediction, evaluation and mechanism of action of traditional Chinese medicine on the information level, and realize traditional Chinese medicine based on data The transformation of modernization has expanded the field of application of traditional Chinese medicine and the development of new drugs.

The key to the evaluation of the efficacy of traditional Chinese medicine is how to establish and determine the active molecules of traditional Chinese medicine, the best combination of active molecules, the reasonable matching of targets, and the correlation between symptoms and drug efficacy. The theory of the efficacy component of traditional Chinese medicine believes that the efficacy of traditional Chinese medicine is based on the efficacy component, which can explain the correlation between the efficacy and substance, substance and quality, quality and clinical efficacy of traditional Chinese medicine ${ }^{[52]}$. Through the rapid screening by computer, the effective component group of traditional Chinese medicine can be obtained in the shortest time, thus providing a shortcut for the next step. Only the molecules that overcome the ADME/T barrier in the traditional Chinese medicine component are expected to exert their efficacy, and only these molecules can become effective component candidate molecules ${ }^{[53,54]}$. The proposal of QSP will provide a huge opportunity for the modern development of Chinese medicine. The original intention of QSP in the research of traditional Chinese medicine is to develop a mathematical and computational model 


\section{APPLICATION VALUE OF QUANTITATIVE SYSTEM PHARMACOLOGY IN DRUG DISCOVERY}

FOR TRADITIONAL CHINESE MEDICINE

that is suitable for the research of complex systems of traditional Chinese medicine and integrates several time-space data, and to establish multiple elements (drug molecules, targets, cells, tissues, organs, etc.) within the model. Interactions to clarify and predict drug efficacy and toxicity. And finally build a multi-dimensional model that spans the differences in knowledge among molecules, cells, tissues, and patients, and implements pre-clinical and clinical research systematic theories and methods for evaluating target screening and testing to verify treatment ideas.

QSP provides feasible theories and ideas for studying traditional Chinese medicine prescriptions at an overall level. It not only can establish a "combination of disease and syndrome" model, but also emphasizes "correspondence of prescription and syndrome". In addition, its scientific nature is also reflected in the toxicology research of traditional Chinese medicine (especially toxic traditional Chinese medicine). Combined with the research of systems biology, $\mathrm{Lu} \mathrm{Yao}{ }^{[55]}$ once proposed the concept of "certification", that is, "certification" is an organic and comprehensive functional state, which is composed of a regulatory center and its many molecular networks, and serves as a The basis of response and self-regulation. Carrying out QSP research on traditional Chinese medicine: First of all, it is necessary to clarify the absorption, distribution, metabolism, excretion and toxicity properties of traditional Chinese medicine ingredients in the human body. After that, it is necessary to carry out network target modeling and network pharmacological analysis modeling to break the "black box operation" of traditional Chinese medicine research, and to find the best combination of synergistic and detoxifying effects of Chinese herbal compound for basic research of Chinese herbal compound and determine The main components or active substances of the compound, as well as the determination of the optimal ratio of the drug, provide the basis ${ }^{[56,57]}$.

At present, the application of QSP in the development of Chinese medicine is very limited. In addition to some changes faced by the domestic pharmaceutical industry in China, the National Drug Administration (CNDA) has issued a series of guidelines, some of which are related to quantitative pharmacology ${ }^{[58,59]}$, some recommend the use of quantitative pharmacology ${ }^{[60-66]}$. Limited to my country's quantitative pharmacology research started late, Chinese medicine as the cultural essence and treasure in the long history of my country, because of its complex and huge systemic nature, the same research case is not much. Fang and other teams have done a series of studies on quantitative and systemic pharmacology ${ }^{[67-70]}$, in which quantitative and systematic pharmacology provided a powerful tool for the development of new tumortargeted therapies for natural product drugs ${ }^{[67]}$ and aging-related diseases ${ }^{[69]}$.The methods and tools of the computing system pharmacology. In short, they first developed a computer system pharmacology framework for systematically predicting drug-target interactions of natural products, and then established multiple prediction network models and statistical network models with high accuracy. It is proved that the drug-target interaction predicted by calculation and experimental verification can overcome the data incompleteness problem of the currently available experimental data. This research can provide a good reference for QSP research of traditional Chinese medicine.

Currently, the application of QSP in tumor immunotherapy ${ }^{[71-75]}$, Alzheimer's disease ${ }^{[76-79]}$ and other diseases has relatively mature and successful models. Based on the tips of QSP design, evaluation, modeling and experimental verification in the literature ${ }^{[49,80-84]}$, combined with the characteristics of Chinese medicine itself systemic and network, the following research strategies for QSP research of Chinese medicine are proposed: Public database and experimental means to build a database of the overall chemical composition of single medicine or compound Chinese medicine. (2) Candidate chemical components were obtained through ADME/T screening and experimental verification. (3) Take the candidate chemical components as the object, and obtain the network and system targets through the drug targeting technology. (4) Carry out correlation analysis of network structure, key targets and disease symptoms and network static and dynamic analysis of drug disturbance. (5) Verify the above results with each other and carry out analysis of Founder 
correlation, symptom correlation and drug action mechanism. (6) According to the characteristics of traditional Chinese medicine and diseases, parameter design, evaluation and optimization are carried out to establish a QSP model. (7) Obtain quantitative analysis results based on ADME/T, target network and overall pharmacokinetics to clarify the QSP mechanism of traditional Chinese medicine. (8) The QSP model is continuously calibrated and iteratively updated based on the overall data of cells, tissues, organs, and clinical data, and finally obtains excellent traditional Chinese medicine prescription.

\section{4 | OUTLOOK:}

The main obstacles in the current research on traditional Chinese medicine are unclear material basis and vague mechanism of action. The diversity of its components, the uncertainty of targets, and the complex interactions between drugs and targets make the research of traditional Chinese medicine face great difficulties. Because of its huge and complicated system, traditional Chinese medicine is difficult to achieve systematic, complete and scientific evaluation simply by using traditional experimental methods. Obviously, the best way to solve complex problems is the intervention of computer technology. In recent years, there has been a surge in research interest in QSP as a mechanism to provide information for drug development. The current situation of drug development and the experimental evidencedriven QSP model, although there are still different voices, it is undeniable that QSP has a great potential in drug development and guiding regulatory decisions. QSP can be used to generate hypotheses and support quantitative understanding of new mechanisms of action in the context of specific tissues, diseases or non-clinical experiments or clinical patient populations. QSP can be further used to optimize dosages and dosage regimens or to support drug combination dosage decisions The early intervention and full participation of QSP in the development of traditional Chinese medicine can form a model-led drug development model to improve the efficiency of research and development and scientific review, reduce the cost of research and development, and shorten the time to market for new drugs. We hope that the QSP model can break through the inherent bottleneck of Chinese medicine research. Simply put, a common vision is to quickly and steadily move Chinese medicine to the international stage through the QSP method and make its due to contribution to the prevention and treatment of human diseases. This study first distinguishes between QSP and other quantitative pharmacology, secondly raises the problems faced by the research on the characteristics of traditional Chinese medicine itself, and then summarizes the role of QSP in the research in traditional Chinese medicine and research strategies, in order to provide reference for the study of traditional Chinese medicine and new ideas.

\section{Authors' Contributions}

Fuxue Meng disigned this study and prepared the manuscript, Xiaomai Tao researched literature and data analysis, Fuxue Meng evaluated the quality of this study and agree to the final version.

\section{Conflicts of Interests}

The authors have no conflicts of interests.

\section{Funding}

This work was supported by no funding.

\section{5 | REFERENCES:}

[1] Ogishima S. Human Genome Data and Drug Development. Gan To Kagaku Ryoho. 2018 45(4): 597-600.

[2] Lum PY, Derry JM, Schadt EE. Integrative genomics and drug development. Pharmacogenomics. 2009 10(2): 203-212.

[3] Chou KC. Some illuminating remarks on molecular genetics and genomics as well as drug development. Mol Genet Genomics. 2020 295(2): 261-274.

[4] Dugger SA, Platt A, Goldstein DB. Drug development in the era of precision medicine. Nat Rev Drug Discov. 2018; 17(3):183-196.

[5] Lyu J, Wang S, Balius TE, Singh I, Levit A, Moroz YS, O'Meara MJ, Che T, Algaa E, Tolmachova K, Tolmachev AA, Shoichet BK, Roth BL, Irwin JJ. Ultra-large library docking for discovering new 

FOR TRADITIONAL CHINESE MEDICINE

chemotypes. Nature. 2019; 566, 224-229.

[6] Wang YH, Yang L. Modern Chinese Medicine Research System Based on System Pharmacology. World Chinese Medicine. 2013 8(7): 801-808.

[7] Gibbs JP, Menon R, Kasichayanula S. Bedside to Bench: Integrating Quantitative Clinical Pharmacology and Reverse Translation to Optimize Drug Development. Clin Pharmacol Ther. 2018; 103(2):196198.

[8] Geerts H, Gieschke R, Peck R. Use of quantitative clinical pharmacology to improve early clinical development success in neurodegenerative diseases. Expert Rev Clin Pharmacol. 2018; 11(8):789-795.

[9] EFPIA MID3 Workgroup, Marshall SF, Burghaus R, Cosson V, Cheung SY, Chenel M, DellaPasqua O, Frey N, Hamrén B, Harnisch L, Ivanow F, Kerbusch T, Lippert J, Milligan PA, Rohou S, Staab A, Steimer JL, Tornøe C, Visser SA. Good Practices in Model-Informed Drug Discovery and Development: Practice, Application, and Documentation. CPT Pharmacometrics Syst Pharmacol. 2016; 5(3):93-122.

[10] Hardy T, Abu-Raddad E, Porksen N, De Gaetano A. Evaluation of a mathematical model of diabetes progression against observations in the Diabetes Prevention Program. Am J Physiol Endocrinol Metab. 2012; 303(2):E200-212.

[11] Messinis DE, Melas IN, Hur J, Varshney $\mathrm{N}$, Alexopoulos LG, Bai JPF. Translational systems pharmacology-based predictive assessment of drug-induced cardiomyopathy. CPT Pharmacometrics Syst Pharmacol. 2018; 7(3):166-174.

[12] Nijsen M, Wu F, Bansal L, Bradshaw-Pierce E, Chan JR, Liederer BM. Preclinical QSP modeling in the pharmaceutical industry: an IQ consortium survey examining the current landscape. CPT Pharmacometrics Syst Pharmacol. 2018; 7(3):135-146.

[13] Rowland M, Peck C, Tucker G. Physiologicallybased pharmacokinetics in drug development and regulatory science. Annu Rev Pharmacol Toxicol. 2011 51: 45-73.

[14] Mould DR. Model-based meta-analysis: an important tool for making quantitative decisions during drug development. Clin Pharmacol Ther. 2012 92(3) : 283-286.
[15] Vicini P, van der Graaf PH. Systems pharmacology for drug discovery and development: paradigm shift or flash in the pan. Clin Pharmacol Ther, 2013, 93 (5) : 379-381.

[16] Dayneka NL, Garg V, Jusko WJ. Comparison of four basic models of indirect pharmacodynamic responses. J Pharmacokinet Biopharm. 1993 21(4) : 457-478.

[17] Sheiner LB. Learning versus confirming in clinical drug development. Clin Pharmacol Ther. 1997 61 (3) : 275-291.

[18] EMA. Guideline on the qualification and reporting of physiologically based pharmacokinetic ( PBPK) modelling and simulation [EB/OL]. [20167-29].

http://www.ema.europa.eu/docs/en-GB/document-li brary/Scientific-guideline/2016 /07/WC500211315. pdf.

[19] FDA. Physiologically based pharmacokinetic analyses- format and content guidance for industry [EB/OL]. [2016-12-01]. https://www.fda.gov/downl oads/drugs/guidancecomplianceregulatoryinformation /guid- ances/ucm531207.pdf.

[20] Ke A, Barter Z, Rowland-Yeo K, Almond L. Towards a Best Practice Approach in PBPK Modeling: Case Example of Developing a Unified Efavirenz Model Accounting for Induction of CYPs 3A4 and 2B6. CPT Pharmacometrics Syst Pharmacol. 2016;5(7):367-76.

[21] Zhao P. Report from EMA workshop on qualification and reporting of physiologically-based pharmacokinetic (PBPK) modelling and simulation. CPT Pharmacometrics Syst Pharmacol, 2017, 6(2) : 7172.

[22] van der Graaf PH, Benson N. The role of quantitative systems pharmacology in the design of fifirst-in-human trials. Clin Pharmacol Ther. 2018; 104(5):797.

[23] Liu DY, Wang K, Ma GL, Xiang XQ, Liu J, Zhao $\mathrm{P}$. The value and general considerations of quantitative harmacology research in ew drug development. Chinese Clin Pharmacology and Therapeutics. 2018 23(9): 961-973.

[24] Chen B, Dong JQ, Pan WJ, Ruiz A. Pharmacokinetics/pharmacodynamics model-supported 


\section{MANUSCRIPT CENTRAL}

early drug development. Curr Pharm Biotechnol. 2012;13(7):1360-1375.

[25] Lavé T, Caruso A, Parrott N, Walz A. Translational $\mathrm{PK} / \mathrm{PD}$ modeling to increase probability of success in drug discovery and early development. Drug Discov Today Technol. 2016; 21-22:27-34.

[26] Wong H, Bohnert T, Damian-Iordache V, et al. Translational pharmacokinetic-pharmacodynamic analysis in the pharmaceutical industry: an IQ Consortium PK-PD Discussion Group perspective. Drug Discov Today. 2017;22(10):1447-1459.

[27] Sorger PK, Altman RB, Brouwer KLR, Califano A, D'Argenio DZ. Quantitative and Systems Pharmacology in the Post-genomic Era: New Approaches to Discovering Drugs and Understanding Therapeutic Mechanisms. 2011.

[28]Bradshaw EL, Spilker ME, Zang R, Bansal L, He H, Jones RDO, Le K, Penney M, Schuck E, Topp B, Tsai A, Xu C, Nijsen MJMA, Chan JR. Applications of Quantitative Systems Pharmacology in ModelInformed Drug Discovery: Perspective on Impact and Opportunities. CPT Pharmacometrics Syst Pharmacol. 2019; 8(11):777-791.

[29] Richiardi J, Altmann A, Milazzo A-C, Chang C, Chakravarty MM, Banaschewski T, Barker GJ, Bokde ALW, Bromberg U, Buchel C, Conrod P, Fauth-Buhler M, Flor H, Frouin V, Gallinat J, Garavan $H$, Gowland $P$, Heinz A, Lemaitre $H$, Mann KF, Martinot J-L, Nees F, Paus T, Pausova Z, Rietschel M, Robbins TW, Smolka MN, Spanagel R, Strohle A, Schumann G, Hawrylycz M, Poline J-B, Greicius MD. Correlated gene expression supports synchronous activity in brain networks. Science.2015; 348:1241-1244.

[30] Chen R, Wang J, Zhan R, Zhang L, Wang X. Integrated Systems Pharmacology, Urinary Metabonomics, and Quantitative Real-Time PCR Analysis to Uncover Targets and Metabolic Pathways of the You-Gui Pill in Treating Kidney-Yang Deficiency Syndrome. Int J Mol Sci. 2019;20(15):3655.

[31]Ruiz-Cerdá ML, Irurzun-Arana I, GonzálezGarcia I, Hu C, Zhou H, Vermeulen A, Trocóniz IF, Gómez-Mantilla JD. Towards patient stratification and treatment in the autoimmune disease lupus erythematosus using a systems pharmacology approach.
Eur J Pharm Sci. 2016; 94:46-58.

[32] Fleisher B, Brown AN, Ait-Oudhia S. Application of pharmacometrics and quantitative systems pharmacology to cancer therapy: The example of luminal a breast cancer. Pharmacol Res. 2017;124:2033.

[33] Stites EC, Shaw AS. Quantitative Systems Pharmacology Analysis of KRAS G12C Covalent Inhibitors. CPT Pharmacometrics Syst Pharmacol. 2018; 7(5):342-351.

[34] Geerts H, Wikswo J, van der Graaf PH, Bai JPF, Gaiteri C, Bennett D, Swalley SE, Schuck E, Kaddurah-Daouk R, Tsaioun K, Pelleymounter M. Quantitative Systems Pharmacology for Neuroscience Drug Discovery and Development: Current Status, Opportunities, and Challenges. CPT Pharmacometrics Syst Pharmacol. 2020; 9(1):5-20.

[35] Gobburu JV. Pharmacometrics 2020. J Clin Pharmacol, 2010; 50(9 Suppl): 151S-157S.

[36] Yildirim MA, Goh KI, Cusick ME, Barabási AL, Vidal M. Drug-target network. Nat Biotechnol. 2007; 25(10):1119-1126.

[37] Yu H, Chen J, Xu X, Li Y, Zhao H, Fang Y, Li X, Zhou W, Wang W, Wang Y. A systematic prediction of multiple drug-target interactions from chemical, genomic, and pharmacological data. PLoS One. 2012;7(5):e37608.

[38] Wang YY. System Biology and the Development of Chinese Medicine-The Relationship between System Theory and Reduction Theory in Chinese Medicine Research. Word Sci Technol Mod Tradit Chin Med. 2007; 9(1): 70-73,79.

[39] Zhao YJ, Li F, Shi BB, Li Y. Pharmacology of Traditional Chinese Medicine System II: Application Progress in Drug Development and Compound Research. Liaoning Journal of Traditional Chinese Medicine. 2020; 47(3): 213-220

[40] Zhang RF, Song XY. Survey of pharmacokinetics research of traditional Chinese medicine. Chinese and Foreign Health Digest. 2013 (27): 645-649

[41] Xing PL, Liu CS. Analysis on the bottleneck of Chinese medicine development and its development trend. Daqing She Hui Ke Xue. 2012; (1):88-89. 

FOR TRADITIONAL CHINESE MEDICINE

[42] Qiu J. 'Back to the future' for Chinese herbal medicines. Nature reviews. Drug discovery. 2007; (6):506-507.

[43] Verpoorte R, Crommelin D, Danhof M, Gilissen LJ, Schuitmaker LF, vander Greef J, Witkamp RF. Commentary: A systems view on the future of medicine: inspiration from Chinese medicine?. Journal of ethnopharmacology. 2009; 121(3): 479481.

[44] Ke-Yong N, Min L. Application of morbid animal model in drug safety evaluation of traditional Chinese medicine. Front Pharmacol. 2015; 6:37

[45] Li L, Liu JX, Ren JX, Guo H, Lin CR. Zhongguo Zhong Yao Za Zhi. 2017; 42(2):254-258.

[46] Chen JC, Liu JX, Lin CR, Ren JG, Li L, Guo H. Zhongguo Zhong Yao Za Zhi. 2018; 43(11):21772183.

[47] Zhang HQ. The development direction of Chinese medicine. Zhongguo Min Zu Min Jian Yi Yao. 2010; 4: 6-7

[48] Wu PX, Wang XX, Liu Yan. Comparison between Syndrome Differentiation and Treatment of Traditional Chinese Medicine and Precision Medicine. She Jie Ke Xue Ji Shu (Zhong Yi Yao Xian Dai Hua). 2016; 18(8):1219-1223.

[49] Wang YH, Li Y. Systematic pharmacology principle method and application. Da Lian Li Gong University Press. 2016. 9

[50] Shu Z, Wu T, Shahen M, Guo Z, Shu J, Wang HE, Shar AH, Farag MR, Alagawany M, El-Hack MEA, Liu C. System-Pharmacology Dissection of Traditional Chinese herbs SINI Decoction for Treatment of Cardiovascular Diseases. An Acad Bras Cienc. 2019 ;91(3):e20180424.

[51] Wang D, Gu J, Zhu W, Luo F, Chen L, Xu X, Lu C. PDTCM: a systems pharmacology platform of traditional Chinese medicine for psoriasis. Ann Med. 2017;49(8):652-660.

[52] Pan GF, Zhu XX, Zhang XD. Studies on System Biology and Effective Components of Traditional Chinese Medicine. She Jie Ke Xue Ji Shu (Zhong Yi Yao Xian Dai Hua). 2008,10(2):5-11.

[53] Hao DC, Xiao PG. Impact of Drug Metabolism/Pharmacokinetics and their Relevance
Upon Traditional Medicine-based Cardiovascular Drug Research. Curr Drug Metab. 2019; 20(7):556574.

[54] Hao DC, Ge GB, Xiao PG, Wang P, Yang L. Drug metabolism and pharmacokinetic diversity of ranunculaceae medicinal compounds. Curr Drug Metab. 2015;16(4):294-321.

[55] LuY, Liu B, Ji N. New Ideas on Basic Research of Traditional Chinese Medicine-System Biology. Chinese Med Clin Res. 2010 2(19): 108-109.

[56] Li Z, Liu J, Zhang D, Du X, Han L, Lv C, Li Y, Wang R, Wang B, Huang Y. Nuciferine and paeoniflorin can be quality markers of Tangzhiqing tablet, a Chinese traditional patent medicine, based on the qualitative, quantitative and dose-exposureresponse analysis. Phytomedicine. 2018;44:155-163.

[57] Zhang X, Liu T, Fan X, Ai N. In silico modeling on ADME properties of natural products: Classification models for blood-brain barrier permeability, its application to traditional Chinese medicine and in vitro experimental validation. J Mol Graph Model. 2017;75:347-354.

[58] CFDA. Technical guidelines for clinical trial data management [EB/OL]. [2016-7-27]. http: //ww w.cde.org.cn /zdyz. do? method $=$ largePage\&id $=$ 271.

[59] CFDA. Guiding rinciples for planning and reporting of drug clinical trial data management and statistical analysis [EB/OL]. [2016-7-27]. http:// w ww.cde.org.cn /zdyz.do? method =largePage\&id = 270 .

[60] CFDA. General considerations for drug clinical trials [EB/OL].[2017-1-18]. http: //www.cde.org.cn /zdyz. do? method = largePage\&id $=263$.

[61] CFDA.Guiding principles for pharmacokinetic research techniques in patients with liver damage[EB/OL].[2012-5-15]. http: //www. Cde.org.cn /zdyz. do? method $=$ largePage\&id $=$ 150.

[62] CFDA.Guiding principles for pharmacokinetic research techniques in patients with renal impairment [EB/OL].[2012-5-15]. http://www.cde.org.cn /zdyz.do? method $=$ largePage\&id $=145$.

[63] CFDA.Guiding principles for drug interaction studies [EB/OL].[2012-5-15]. http: //www.cde. Org. 


\section{MANUSCRIPT CENTRAL}

cn $/$ zdyz. do method $=$ largePage \&id $=147$.

[64] CFDA.Technical guidelines for extrapolation of medication data to the pediatric population [EB/OL]. [2017-5-18]. http: //www.cde.org.cn/zdyz .do? method $=$ largePage\&id $=262$.

[65] CFDA. Guiding principles for pharmacokinetic research techniques in pediatric population $[\mathrm{EB} / \mathrm{OL}]$. [2014-7-11]. http://www.cde.org.cn/zdyz.do?metho $\mathrm{d}=$ largePage \&id $=241$.

[66] CFDA. Guiding Principles for Research on Antimicrobial Breakpoints (Draft for Online Comment) [EB/OL]. [2017-8-18]. http://www. Cde.org. $\mathrm{cn} /$ search.do? method $=$ searchTitle.

[67] Fang J, Wu Z, Cai C, Wang Q, Tang Y, Cheng F. Quantitative and Systems Pharmacology. 1. In Silico Prediction of Drug-Target Interactions of Natural Products Enables New Targeted Cancer Therapy. J Chem Inf Model. 2017; 57(11):2657-2671.

[68] Wu Z, Lu W, Yu W, Wang T, Li W, Liu G, Zhang H, Pang X, Huang J, Liu M, Cheng F, Tang Y. Quantitative and systems pharmacology 2. In silico polypharmacology of $G$ protein-coupled receptor ligands via network-based approaches. Pharmacol Res. 2018; 129:400-413.

[69] Fang J, Gao L, Ma H, Wu Q, Wu T, Wu J, Wang Q, Cheng F. Quantitative and Systems Pharmacology 3. Network-Based Identification of New Targets for Natural Products Enables Potential Uses in Aging-Associated Disorders. Front Pharmacol. 2017;8:747.

[70] Fang J, Cai C, Chai Y, Zhou J, Huang Y, Gao L, Wang Q, Cheng F. Quantitative and systems pharmacology 4. Network-based analysis of drug pleiotropy on coronary artery disease. Eur J Med Chem. 2019;161:192-204.

[71] Milberg O, Gong C, Jafarnejad M, Bartelink IH, Wang B, Vicini P, Narwal R, Roskos L, Popel AS. A QSP Model for Predicting Clinical Responses to Monotherapy, Combination and Sequential Therapy Following CTLA-4, PD-1, and PD-L1 Checkpoint Blockade. Sci Rep. 2019;9(1):11286.

[72] Stroh M, Sagert J, Burke JM, Apgar JF, Lin L, Millard BL, Michael Kavanaugh W. Quantitative Systems Pharmacology Model of a Masked, Tumor-Activated Antibody. CPT Pharmacometrics
Syst Pharmacol. 2019; 8(9):676-684.

[73] Wang H, Milberg O, Bartelink IH, Vicini P, Wang B, Narwal R, Roskos L, Santa-Maria CA, Popel AS. In silico simulation of a clinical trial with anti-CTLA-4 and anti-PD-L1 immunotherapies in metastatic breast cancer using a systems pharmacology model. R Soc Open Sci. 2019; 6(5):190366.

[74] Betts A, Haddish-Berhane N, Shah DK, van der Graaf PH, Barletta F, King L, Clark T, Kamperschroer C, Root A, Hooper A, Chen X. A Translational Quantitative Systems Pharmacology Model for CD3 Bispecific Molecules: Application to Quantify $\mathrm{T}$ Cell-Mediated Tumor Cell Killing by PCadherin LP DART ${ }^{\circledR}$. AAPS J. 2019; 21(4):66.

[75] Hardiansyah D, Ng CM. Quantitative Systems Pharmacology Model of Chimeric Antigen Receptor T-Cell Therapy. Clin Transl Sci. 2019;12(4):343349.

[76] Hampel H, Lista S, Mango D, Nisticò R, Perry G, Avila J, Hernandez F, Geerts H, Vergallo A; Alzheimer Precision Medicine Initiative (APMI). Lithium as a Treatment for Alzheimer's Disease: The Systems Pharmacology Perspective. J Alzheimers Dis. 2019; 69(3):615-629.

[77] Clausznitzer D, Pichardo-Almarza C, Relo AL, van Bergeijk J, van der Kam E, Laplanche L, Benson N, Nijsen M. Quantitative Systems Pharmacology Model for Alzheimer Disease Indicates Targeting Sphingolipid Dysregulation as Potential Treatment Option. CPT Pharmacometrics Syst Pharmacol. 2018; 7(11):759-770.

[78] Geerts H, Spiros A, Roberts P. Impact of amyloid-beta changes on cognitive outcomes in Alzheimer's disease: analysis of clinical trials using a quantitative systems pharmacology model. Alzheimers Res Ther. 2018;10(1):14-21.

[79] Geerts H, Spiros A, Roberts P, Carr R. Towards the virtual human patient. Quantitative Systems Pharmacology in Alzheimer's disease. Eur J Pharmacol. 2017; 817:38-45.

[80] Helmlinger G, Sokolov V, Peskov K, Hallow KM, Kosinsky Y, Voronova V, Chu L, Yakovleva T, Azarov I, Kaschek D, Dolgun A, Schmidt H, Boulton DW, Penland RC. Quantitative Systems Pharmacology: An Exemplar Model-Building Workflow With 


\section{FOR TRADITIONAL CHINESE MEDICINE}

Applications in Cardiovascular, Metabolic, and Oncology Drug Development. CPT Pharmacometrics Syst Pharmacol. 2019; 8(6):380-395.

[81] Gaillard-Bigot F, Zendjidjian XY, Kheloufi F, Casse-Perrot C, Guilhaumou R, Micallef J, Fakra E, Azorin JM, Blin O. Quantitative System Pharmacology (QSP): An Integrative Framework for paradigm change in the treatment of the first-episode schizophrenia. Encephale. 2018; 44(6S):S34-S38.

[82] Traynard P, Tobalina L, Eduati F, Calzone L, Saez-Rodriguez J. Logic Modeling in Quantitative Systems Pharmacology. CPT Pharmacometrics Syst Pharmacol. 2017; 6(8):499-511.

[83] Ribba B, Grimm HP, Agoram B, Davies MR, Gadkar K, Niederer S, van Riel N, Timmis J, van der Graaf PH. Methodologies for Quantitative Systems
Pharmacology (QSP) Models: Design and Estimation. CPT Pharmacometrics Syst Pharmacol. 2017; 6(8):496-498.

[84] Elmokadem A, Riggs MM, Baron KT. Quantitative Systems Pharmacology and PhysiologicallyBased Pharmacokinetic Modeling With mrgsolve: A Hands-On Tutorial. CPT Pharmacometrics Syst Pharmacol. 2019;8(12):883-893.

How to cite this article: Meng F., Tao X. Application value of quantitative system pharmacology in drug discovery for traditional Chinese medicine. Journal of Medical Care Research and Review. 2020;425-436. https://doi.org/ $10.15520 /$ mcrr.v3i9. 113 